\title{
Multiparameter Elastic Full Waveform Inversion With Facies Constraints
}

Zhen-dong Zhang*, Tariq Alkhalifah* and Ehsan Zabihi Naeini ${ }^{\dagger}$

${ }^{*}$ King Abdullah University of Science and Technology.

${ }^{\dagger}$ Ikon Science.

\section{SUMMARY}

Full waveform inversion (FWI) aims fully benefit from all the data characteristics to estimate the parameters describing the assumed physics of the subsurface. However, current efforts to utilize full waveform inversion as a tool beyond acoustic imaging applications, for example in reservoir analysis, faces inherent challenges related to the limited resolution and the potential trade-off between the elastic model parameters. Adding rock physics constraints does help to mitigate these issues, but current approaches to add such constraints are based on including them as a priori knowledge mostly valid around the well or as a boundary condition for the whole area. Since certain rock formations inside the Earth admit consistent elastic properties and relative values of elastic and anisotropic parameters (facies), utilizing such localized facies information in FWI can improve the resolution of inverted parameters. We propose a novel confidence map based approach to utilize the faciesbased constraints in both isotropic and anisotropic elastic FWI. We invert for such a confidence map using Bayesian theory, in which the confidence map is updated at each iteration of the inversion using both the inverted models and a prior information. The numerical examples show that the proposed method can reduce the trade-offs and also can improve the resolution of the inverted elastic and anisotropic properties.

\section{INTRODUCTION}

Uncertainties in inversion results are usually related to the forward modeling engine and the assumptions behind it (Tarantola, 2005). For example, we assume that the uncertainties in the observed data have normal distributions and have nothing to do with such uncertainties. However, the uncertainties caused by simulation engine can be reduced by considering a more accurate physics to describe the earth response. To better simulate wave propagation, depending on the efficiency and accuracy requirements, elastic and anisotropic elastic wave equations are used as the forward modeling engine in full waveform inversion. With the current improvements in computational power, solving these complex equations is becoming more and more practical. However, more complex physics require more parameters to describe the real earth and inevitably introduces more null space.

Estimating elastic and anisotropic parameters is an ongoing topic of interest for the seismic exploration community of the seismic exploration community. In current practice, due to an inherent trade-off between parameters, for example, P-wave velocity and density, the density model is not usually updated at all to reduce the nonlinearity (null space) of the inversion
(Köhn et al., 2012). However, to get a better understanding of the subsurface, a more accurate multiparameter inversion is necessary. Other common ways to reduce the null space in multiparameter inversion are choice of better parametrization (Operto et al., 2013; Alkhalifah and Plessix, 2014; Oh and Alkhalifah, 2016) and incorporation of a priori information to constrain the inversion. Utilizing a priori information in the form of preconditioning or regularization has been shown to efficiently reduce the null space (Asnaashari et al., 2013).

As opposed to deterministic logic, Bayesian theory provides a natural platform to incorporate available prior information of the model for many geophysical inversion problems (Buland and Omre, 2003). In classic AVO inversion, a more advanced type of constraint, namely per-facies rock physics constraints, has been proved to be very effective in optimizing seismic inversion (Zabihi Naeini and Exley, 2017). Zabihi Naeini et al. (2016) discussed the main components of FWI as a potential reservoir characterization tool and one of their suggestions was to use facies based rock physics constraints in FWI. Later, Zhang et al. (2017) applied a simplified version of Bayesian inversion to classify the facies and then utilize the inverted facies as constraints in isotropic elastic FWI. In this study, we extend the previous work to both isotropic and anisotropic elastic FWI using the full Bayesian inversion framework. We assume that the inverted models adhere to a Gaussian distribution (Tarantola, 2005) and, iteratively, based on the prior information, develop and update a facies confidence map, which in turn is used as a regularization term in the inversion. We test the proposed method on both isotropic and anisotropic elastic models.

\section{THEORY}

\section{Elastic FWI With Regularization}

Our proposed misfit function contains a standard data misfit term, a smoothed Total Variation (TV) regularization term and a facies-based regularization term, as follows

$$
J(\boldsymbol{m})=J_{d}(\boldsymbol{m})+\alpha J_{T V}(\boldsymbol{m})+\beta J_{\text {prior }}(\boldsymbol{m}),
$$

where $\alpha$ and $\beta$ control the contribution from the penalty terms, and $\boldsymbol{m}$ denotes a vector of model parameters.

The standard data misfit is given by

$$
J_{d}=\left\|\mathbf{W}_{d}\left(\boldsymbol{d}^{\text {pre }}-\boldsymbol{d}^{\text {obs }}\right)\right\|^{2},
$$

where $\boldsymbol{d}$, with the corresponding superscripts, denote the vectors of multicomponent data, and $\mathbf{W}_{d}$ is a weighting operator applied to the data, $\mathbf{W}_{d}=\sigma_{d} \mathbf{I}$. Here, $\sigma_{d}$ is the standard deviation of the predicted data. 
We use a smoothed TV as a penalty in the objective function, as follows

$$
J_{T V}=\int \sqrt{\varepsilon^{2}+\|\nabla \boldsymbol{m}\|^{2}} d x
$$

where $\varepsilon$ mitigates the singularities in the gradient.

The last term in equation 1 utilizes an a priori given by the facies constraint, as a penalty, as follows

$$
J_{\text {prior }}=\left\|\mathbf{W}_{m}\left(\boldsymbol{m}^{i n v}-\boldsymbol{m}^{c}\right)\right\|^{2} .
$$

Similarly, $\mathbf{W}_{m}$ is a diagonal matrix, $\boldsymbol{m}^{i n v}$ denotes the inverted model in each iteration, and $\boldsymbol{m}^{c}$ is the calculated confidence map which depends on both the inversion results and the prior information, as we will see below. Usually we only have a general information of the facies without knowing their spatial distributions. Thus, we use Bayesian inversion to estimate their spatial distributions and then use them as constraints in the inversion.

The gradient with respect to the objective function is written as,

$$
\begin{aligned}
\boldsymbol{g}= & \left(\frac{\partial \boldsymbol{d}^{p r e}}{\partial \boldsymbol{m}}\right)^{T} \mathbf{W}_{d}^{T} \mathbf{W}_{d}\left(\boldsymbol{d}^{p r e}-\boldsymbol{d}^{o b s}\right)+ \\
& \alpha \operatorname{div}\left(\frac{\nabla \boldsymbol{m}}{\sqrt{\varepsilon^{2}+\|\nabla \boldsymbol{m}\|^{2}}}\right)+ \\
& \beta \mathbf{W}_{m}^{T} \mathbf{W}_{m}\left(\boldsymbol{m}^{i n v}-\boldsymbol{m}^{c}\right),
\end{aligned}
$$

where the weighting factors $\alpha$ and $\beta$ are decided by several trials.

\section{Bayesian Inversion for Confidence Maps}

Seismic facies are classified based on similarities in any observable attribute of rocks such as elastic properties, connectivity and overall appearance over a geological area. Facies can, therefore, provide rock physics relationships, which can be utilized as constraints in the inversion. This is a key feature (i.e. rock physics constraints per facies) as opposed to assuming only one relationship over the entire area (Kemper and Gunning, 2014; Zabihi Naeini and Exley, 2017).

With a probabilistic type inversion in mind, quantitative FWI can be analyzed from a statistical perspective. Specifically, we use Bayesian inversion to calculate a confidence map from quantitative inversion and a prior knowledge. The first step of Bayesian inversion is to find the maximum likelihood, which is

$$
P(\boldsymbol{f} \mid \boldsymbol{m})=\frac{P(\boldsymbol{f}) \cdot P(\boldsymbol{m} \mid \boldsymbol{f})}{\int P(\boldsymbol{m} \mid \boldsymbol{f}) P(\boldsymbol{f})},
$$

where $\cdot$ denotes element-wise multiplication. $\boldsymbol{m}$ is the vector of inverted model from conventional least-square fitting. $f$ denotes the vector of facies given.

To be consistent with the least-square criterion, the Gaussian model is used to describe the uncertainties in the model space (Tarantola, 2005):

$$
P(\boldsymbol{m} \mid \boldsymbol{f})=\exp (-\gamma(\boldsymbol{m}-\boldsymbol{f}) \odot(\boldsymbol{m}-\boldsymbol{f})),
$$
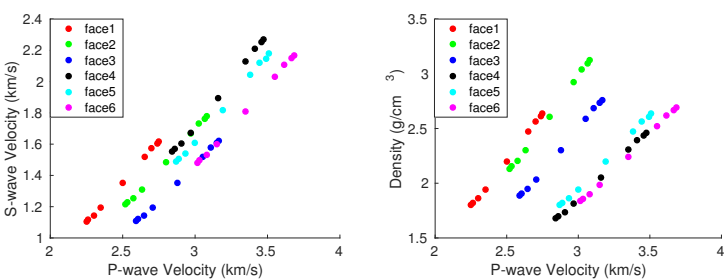

Figure 1: Six facies with normal observation errors. Each color denotes the corresponding group of the face. There are nine observations with normally distributed errors for each face. They're used as the possibility $\mathbf{P}(f)$ in equation 6 .

where $\gamma$ controls the resolution of estimated confidence map. $\odot$ denotes the element-wise multiplication. The solution of the Bayesian inverse problem is then represented by its posterior expectation.

\section{NUMERICAL EXAMPLE}

We will start by testing the approach on an isotropic model, inverting for 3 parameters parameters followed by an anisotropic example with 6 parameters. All the examples are 3D models with a spray of the $2 \mathrm{D}$ slice in the third axis.

\section{Isotropic Example}

We consider a layered model with six facies with nine normally distributed observation errors in each of them as shown in Figure 1. A staggered finite-difference method is used to solve the elastic equation with an absorbing boundary layer condition. The model size is $1.75 \mathrm{~km}$ by $2.5 \mathrm{~km}$ with $50 \mathrm{ex}-$ plosive point sources distributed evenly on the surface shown in Figure 2. The recorded seismic data are multi-component particle velocities. The initial model shown in Figure 3 is a smoothed version of the true model obtained by applying a smoothing operator of length $200 \mathrm{~m}$.
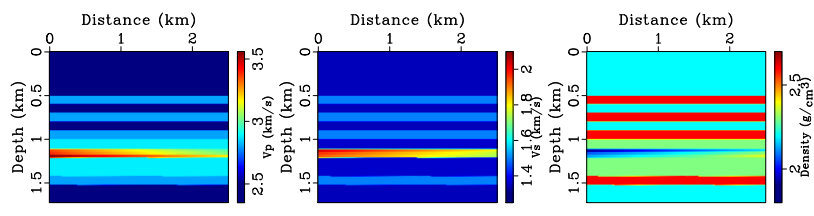

Figure 2: True models. P-wave velocity (left), S-wave velocity (middle) and density (right).
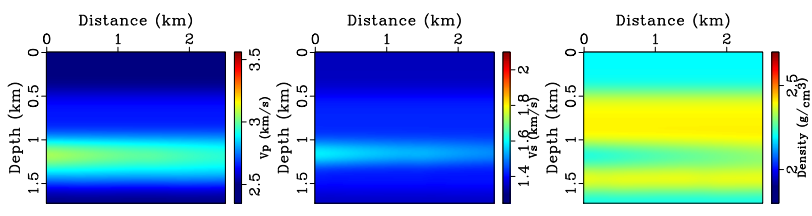

Figure 3: Initial models. P-wave velocity (left), S-wave velocity (middle) and density (right).

Five frequency bands are used in the inversion, which are 2-7 
$\mathrm{Hz}, 2-10 \mathrm{~Hz}, 2-13 \mathrm{~Hz}, 2-16 \mathrm{~Hz}$ and 2-19 Hz, sequentially. We also add random noise to the $2 \mathrm{~Hz}$ low-cut filtered data to form the observed data. We conduct a standard elastic full waveform inversion, for reference, as shown in Figure 4 to compare with the results of our proposed method shown in Figure 5. To assist with the visual comparison, a vertical profile in the middle of the model is also plotted in Figures 6 and 7. The standard method overestimates the $\mathrm{S}$-wave velocities and there is a relatively strong crosstalk between P-wave velocity and density, as expected. However, the proposed facies-based method can recover most of the layers correctly, and at reasonably high resolution. Figure 8 shows the observed data, the predicted data and the data residual for the proposed method at $2-13 \mathrm{~Hz}$ respectively.
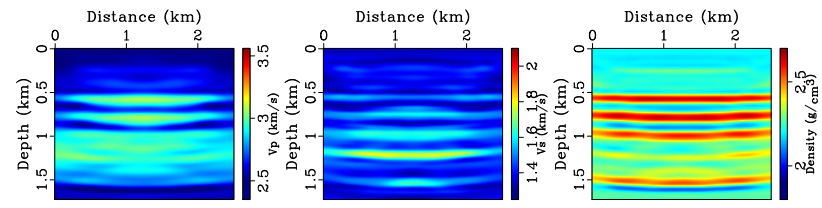

Figure 4: Standard elastic FWI results. P-wave (left), S-wave (middle) velocities and density (right).
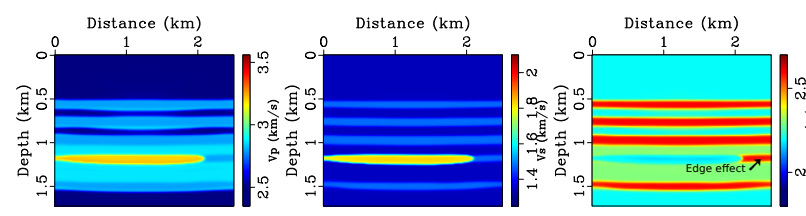

Figure 5: Proposed elastic FWI results. P-wave (left), S-wave (middle) velocities and density (right).
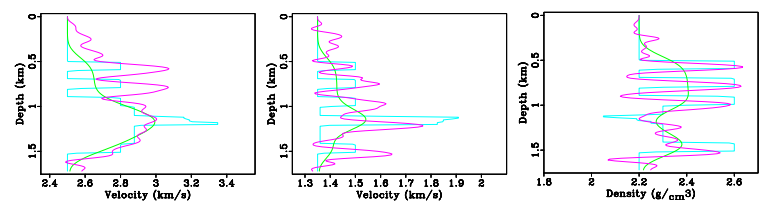

Figure 6: One vertical profile of the conventional method. P-wave (left), S-wave (middle) velocities and density (right). Cyan: true model; Green: initial model; Pink: standard elastic FWI.
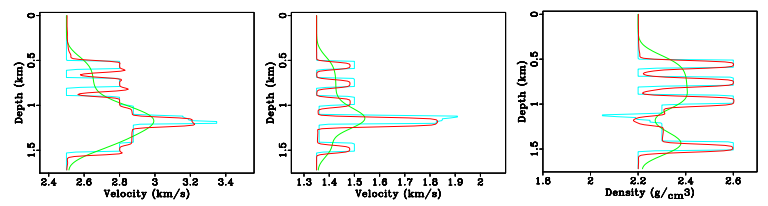

Figure 7: One vertical profile of our proposed method. Pwave velocity (left), S-wave velocity (middle) and density (right). Cyan: true model; Green: initial model; Red: Proposed method.

\section{Anisotropic Example}

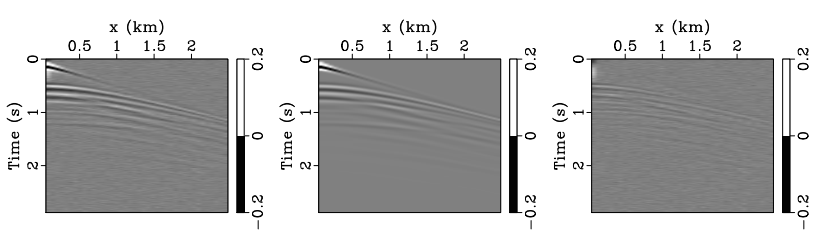

Figure 8: Observed data with random noise at 2-19 Hz (left); Predicted data of the proposed inversion result (middle); Data residual (right).

We extend the isotropic model used in the first example to a transversely isotropic model with a vertical symmetry axis (VTI). The assumed reservoir (at depth $1.2 \mathrm{~km}$ ) is surrounded by VTI layers (from $1 \mathrm{~km}$ to $1.45 \mathrm{~km}$ in depth). Facies are not listed in the example but they are constructed to have a similar behavior (certain relationships with $V_{p}$ ) to the facies used in the first example (e.g. Figure 1). Six parameters $\left(V_{p}, V_{s}\right.$, $\rho, V_{n}, V_{h}$ and $\left.V s_{h}\right)$ are inverted, simultaneously. The configuration of the acquisition system is similar to that of the first example. Four frequency bands, $2-5 \mathrm{~Hz}, 2-7 \mathrm{~Hz}, 2-10 \mathrm{~Hz}$ and $2-16 \mathrm{~Hz}$, are utilized in the multistage approach. Again we add random noise to the observed data. Figure 9 shows the actual model used in the example, note that the potential reservoir is surrounded by VTI layers. The initial model shown in Figure 10 is a smoothed version of the true model, which has the same smoothing radius used in the isotropic case. For comparison, the inverted models from conventional elastic FWI and the proposed method are shown in Figures 11 and 12, respectively. Vertical profiles from the conventional (Figure 13) and the proposed (Figure 14) method inverted models are also plotted. Note that the anisotropic parameters are recovered insufficiently compared to the elastic parameters through the conventional anisotropic elastic FWI approach. However, the proposed statistical approach can improve the inverted anisotropic parameters. The observed data, the predicated data and the corresponding data residual $(2-16 \mathrm{~Hz})$ are shown in Figure 15. The inverted model can predict most of the coherent events in the observed data.

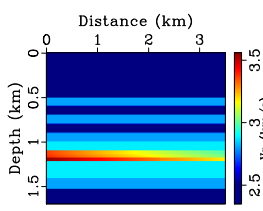

$V_{p}$

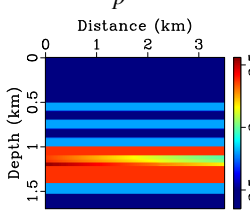

$V_{n}$

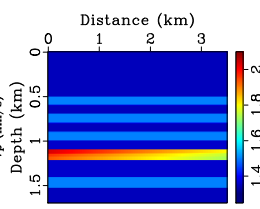

$V_{s}$

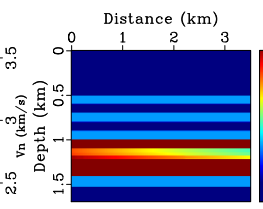

$V_{h}$

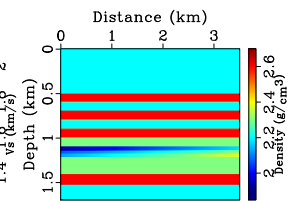

$\rho$

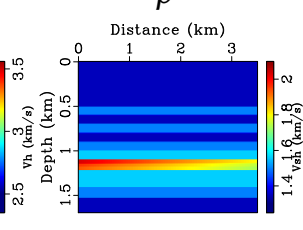

$V s_{h}$
Figure 9: Actual models used in the VTI example. 


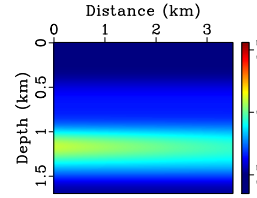

$V_{p}$

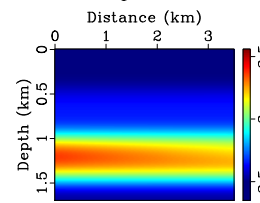

$V_{n}$

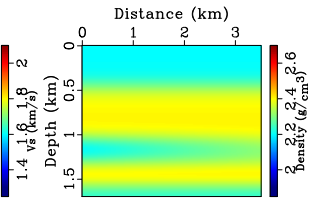

$\rho$

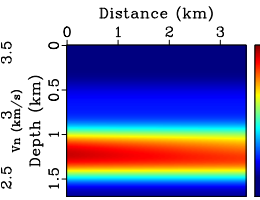

$V_{h}$

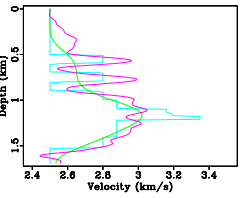

$V_{p}$

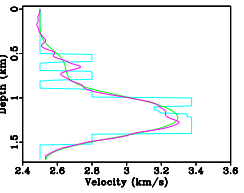

$V_{n}$

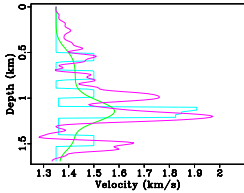

$V_{s}$

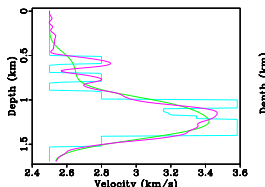

$V_{h}$

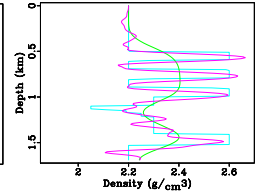

$\rho$

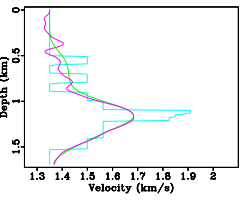

$V s_{h}$

Figure 10: Initial models used in the VTI example.

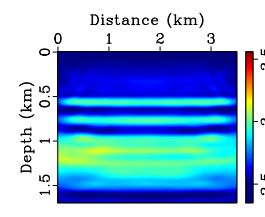

$V_{p}$

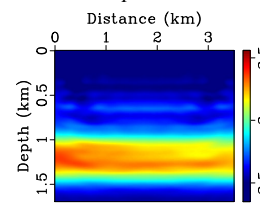

$V_{n}$

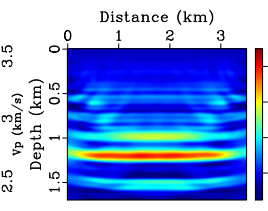

$V_{s}$

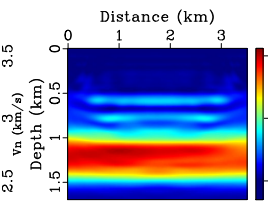

$V_{h}$

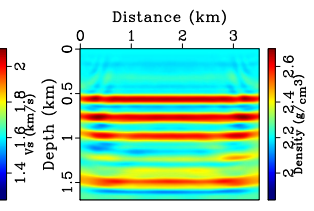

$\rho$

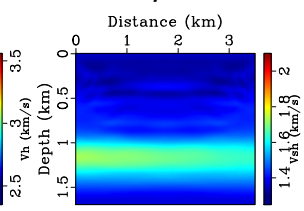

$V s_{h}$

Figure 11: Inverted models from conventional elastic FWI.

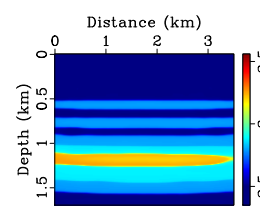

$V_{p}$

Distance (km)

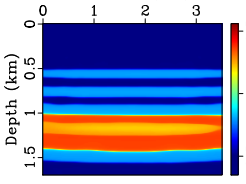

$V_{n}$

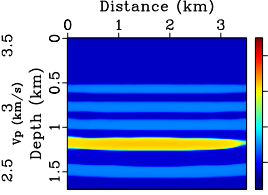

$V_{S}$ Distance $(\mathbf{k m})$

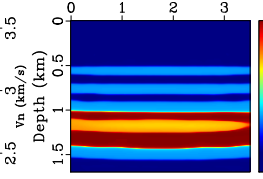

$V_{h}$

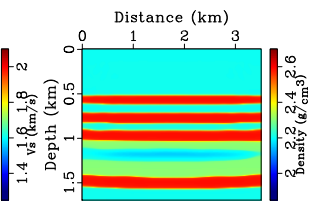

$\rho$

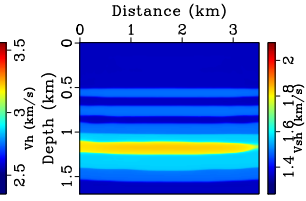

$V s_{h}$

Figure 12: Inverted models from the proposed method.

\section{CONCLUSION}

We proposed a novel approach to utilize facies dependent $a$ prior information to constrain the multiparameter elastic FWI. A statistically driven confidence map is calculated and iteratively updated based on the inversion results and the priors. It is consistent with the framework of the local optimization method, which has the assumption of a Gaussian distribution for both model and data uncertainties. The numerical examples show that the proposed method can suppress the cross-talk

Figure 13: Vertical profiles from the conventional method. Cyan: true models; Green: initial models; Pink: inverted models.
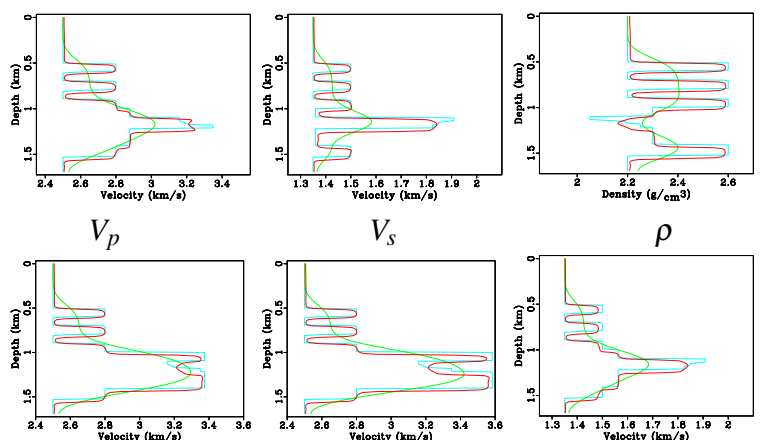

$V_{s}$
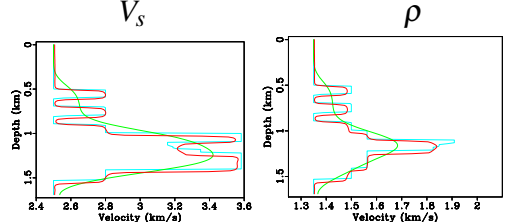

$V_{n}$

$V_{h}$

$V s_{h}$

Figure 14: Vertical profiles from the proposed method. Cyan: true models; Green: initial models; Red: inverted models.
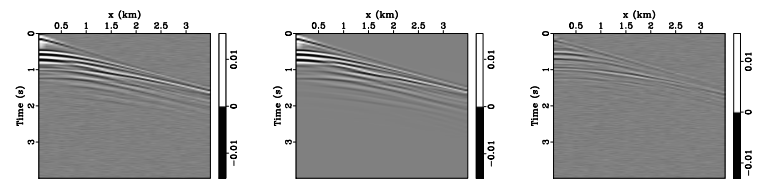

Figure 15: Observed data with random noise at 2-16 Hz (left); Predicted data of the proposed inversion result (middle); Data residual (right).

between different parameters and also can improve the resolution of the estimated anisotropic properties. However, edge effect and other artifacts can degrade the results as the proposed updates depend on the first pass inversion results. The proposed method can be easily incorporated into the general inversion framework.

\section{ACKNOWLEDGMENTS}

We thank Juwon Oh, Bingbing Sun, Vladimir Kazei and Yike Liu (IGG, CAS) for their helpful discussions. For computer time, this research used the resources of the Supercomputing Laboratory at King Abdullah University of Science \& Technology (KAUST) in Thuwal, Saudi Arabia. 
EDITED REFERENCES

Note: This reference list is a copyedited version of the reference list submitted by the author. Reference lists for the 2017 SEG Technical Program Expanded Abstracts have been copyedited so that references provided with the online metadata for each paper will achieve a high degree of linking to cited sources that appear on the Web.

\section{REFERENCES}

Alkhalifah, T., and R.-E. Plessix, 2014, A recipe for practical full-waveform inversion in anisotropic media: An analytical parameter resolution study: Geophysics, 79, no. 3, R91-R101, http://doi.org/10.1190/geo2013-0366.1.

Asnaashari, A., R. Brossier, S. Garambois, F. Audebert, P. Thore, and J. Virieux, 2013, Regularized seismic full waveform inversion with prior model information: Geophysics, 78, no. 2, R25-R36, http://doi.org/10.1190/geo2012-0104.1.

Buland, A., and H. Omre, 2003, Bayesian linearized AVO inversion: Geophysics, 68, 185-198, http://doi.org/10.1190/1.1543206.

Kemper, M., and J. Gunning, 2014, Joint impedance and facies inversion-seismic inversion redefined: First Break, 32, 89-95.

Köhn, D., D. De Nil, A. Kurzmann, A. Przebindowska, and T. Bohlen, 2012, On the influence of model parametrization in elastic full waveform tomography: Geophysical Journal International, 191, 325-345, http://doi.org/10.1111/gji.2012.191.issue-1.

Oh, J.-W., and T. Alkhalifah, 2016, Elastic orthorhombic anisotropic parameter inversion: An analysis of parameterization: Geophysics, 81, no. 6, C279-C293, http://doi.org/10.1190/geo2015-0656.1.

Operto, S., Y. Gholami, V. Prieux, A. Ribodetti, R. Brossier, L. Metivier, and J. Virieux, 2013, A guided tour of multiparameter full-waveform inversion with multicomponent data: From theory to practice: The Leading Edge, 32, 1040-1054, http://doi.org/10.1190/tle32091040.1.

Tarantola, A., 2005, Inverse problem theory and methods for model parameter estimation: SIAM.

Zabihi Naeini, E., T. Alkhalifah, I. Tsvankin, N. Kamath, and J. Cheng, 2016, Main components of fullwaveform inversion for reservoir characterization: First Break, 34, 37-48, http://doi.org/10.3997/1365-2397.2016015.

Zabihi Naeini, E., and R. Exley, 2017, Quantitative interpretation using facies-based seismic inversion: Interpretation, 5, SL1-SL8, http://doi.org/10.1190/INT-2016-0178.1.

Zhang, Z.-D., E. Zabihi Naeini, and T. Alkhalifah, 2017, Facies constrained elastic full waveform inversion: 79th Annual International Conference and Exhibition, EAGE, Extended Abstracts, https://doi.org/10.3997/2214-4609.201700719. 